\title{
Unplanned readmissions in younger and older adult patients: the role of healthcare-related adverse events
}

Fabienne J. H. Magdelijns ${ }^{1 *}$, Larissa Schepers ${ }^{1}$, Evelien Pijpers ${ }^{2}$, Coen D. A. Stehouwer ${ }^{3}$ and Patricia M. Stassen ${ }^{4}$

\begin{abstract}
Background: Readmissions are a burden for patients and increase healthcare costs. In Europe, factors associated with readmissions have not yet been extensively investigated. This study aimed to discover factors associated with readmissions in both young and older adult internal medicine patients. Furthermore, we explored the role of healthcare-related adverse events (AEs) in readmissions.

Methods: All patients admitted through the emergency department to the internal medicine department in the last 2 weeks of each month (2011) were included. Information on index admissions and readmissions, defined as an unplanned admission within 30 days after discharge, was obtained from the electronic patient record system. Demographic, clinical, and organizational factors were evaluated for their association with readmissions.

Results: Of all patients $(n=940), 17.3 \%$ were readmitted; $16.9 \%$ of the younger ( $<65$ years, $n=485)$, and $17.8 \%$ of the older patients ( $\geq 65$ years, $n=455$ ). Dependency in activities of daily living (ADL) was the only factor associated with readmissions in both all ages (OR 2.43) and in older patients (OR 3.19), while age was associated with readmissions in younger patients (OR 1.03 per year). AEs leading to $35.4 \%$ of all index admissions were not associated with readmissions.

Conclusions: Readmissions are common in medical patients, and, thus, remain a reason for concern in terms of patient safety and quality of care. AEs, responsible for to the index admission, were not associated with readmissions. ADL dependency was the only factor associated with readmission in patients of all ages and older patients, indicating that determining which patients are at risk for readmissions is not easy.
\end{abstract}

Keywords: Readmissions, Healthcare-related adverse events, Predictive factors

\section{Background}

Hospital readmissions (defined as an unplanned admission within 30 days after discharge) are not only highly prevalent (11.6-17.5\%) worldwide [1-5], but also a burden to patients, and they increase the already high healthcare costs. In addition, hospital readmissions are seen as a measure of quality of inpatient and post-discharge care $[6,7]$.

\footnotetext{
*Correspondence: fabienne.magdelijns@mumc.nl

${ }^{1}$ Division of General Medicine, Department of Internal Medicine, Maastricht University Medical Centre, Maastricht University, PO Box 5800, 6202 AZ Maastricht, The Netherlands

Full list of author information is available at the end of the article
}

A better understanding of the factors related to readmissions is necessary to develop successful interventions. Some factors, such as race, the use of high-risk medications, comorbidities [Charlson comorbidity index (CCI)], and type of insurance, were found to be associated with readmissions $[1,3,5,8]$. The studies that investigated these factors were mostly performed in the United States, with its specific healthcare structure and healthcare insurance, which make generalizability to settings in Europe difficult. One European study investigated the association between several factors and readmissions in a general medicine department and found that only the factor age (OR 1.01 per year) was independently associated with readmissions 
[9]. Therefore, in particular in Europe, information on factors related to readmissions is scarce.

Since the population is ageing, most hospitalized patients are, or soon will be, old. Older patients more often have comorbidities and disabilities, and they use more medications than younger patients. Multi-morbidity, polypharmacy, and the factor age per se [9] could make older patients more susceptible to hospital readmissions and AEs, which in their turn could lead to hospitalization [10-12]. However, the information on the factors that are associated with readmissions in older patients is as scarce as in patients of all ages and it is unknown whether or not these factors differ between younger and older patients, which we expect.

Readmissions are regarded as healthcare-related adverse events (AEs). It is known that other AEs than readmissions, e.g., medication-related AEs, are a common reason for hospitalization [12-16]. It may be possible that patients who have been hospitalized because of an $\mathrm{AE}$ are particularly vulnerable and thus more susceptible to readmissions. As far as we know, the role of AEs (that have led to hospitalization) in readmissions has not been studied before. In addition, it is unclear whether or not specific factors are associated with readmission in patients who were initially hospitalized because of an AE.

To address the aforementioned gaps in knowledge on factors related to readmissions, we aimed to identify factors, including not formerly investigated factors, such as AEs, which are associated with readmissions within 30 days after discharge in adult medical patients. Furthermore, we investigated which factors were associated with readmissions in younger ( $<65$ years) and older patients ( $\geq 65$ years). In addition, we analysed patients with a first (index) admission because of an AE to reveal factors that are associated with readmissions in this specific subgroup of patients.

\section{Methods}

\section{Setting and study population}

This study was conducted in secondary and tertiary university hospital (Maastricht University Medical Centre; MUMC+) in The Netherlands. Our hospital is a 700-bed teaching hospital, which is the only hospital of the city of Maastricht $(\approx 120,000$ inhabitants) and its surroundings, and which is the only university centre for the province of Limburg $(\approx 1,119,000$ inhabitants $)$. The population of Maastricht is characterized by a high percentage of older patients: $18.1 \%$ are 65 years or older. Annually, 22,000 patients visit our emergency department (ED), which is open $24 \mathrm{~h}, 7$ days a week. In our hospital, all general internal medicine, endocrinology, oncology, haematology, nephrology, gastro-intestinal, and rheumatology patients presenting to the ED are assessed by internists specialized in acute care. General practitioners (GPs) refer the majority of patients (GP service is available $24 \mathrm{~h} / 7$ days). Some patients (notably high urgency patients) arrive by ambulance and a minority of patients are self-presenters. Almost all acutely patients admitted are presented through the ED (for more details on the organization of acute care in The Netherlands, see [17]). Every inhabitant of the Netherlands is obliged to have a health insurance, which ensures accessible healthcare for everyone.

All patients admitted through the ED to the department of internal medicine in the last 2 weeks of each month between January-December 2011 were included. Because of restricted availability of time, we had to limit our inclusion to half a year. By including the last 2 weeks per month of 1 year, we were able to study seasonal influences. Older patients were defined as patients aged 65 years or older, and younger adult patients as patients $<65$ years of age.

The first admission of a patient in our study period was considered the index admission. Exclusion criteria were: (1) (re-)admission to another department than internal medicine or transfer from another hospital, (2) elective or planned (re-)admissions, (3) already included in the study (every patient could only be included once), (4) death inhospital during the index admission, and (5) death within 30 days after discharge unless the patient had already been readmitted prior to death.

\section{Data collection and definitions}

Our hospital has an electronic patient record system, which gave us the opportunity to gather information from both the entire medical and nursing records. Admission charts and discharge letters were used to obtain patients' age, sex, comorbidity, number of medications, living situation and functional status [cognitive function, performance in activities of daily living (ADL) and instrumental activities of daily living (IADL)], reason for admission, length of stay, and day/season of discharge. Comorbidity was calculated using the Charlson comorbidity index (CCI) $[18,19]$. Living situation was categorized as community dwelling or living in a nursing facility. The performance in ADL and IADL was classified as independent or dependent based on information obtained by nurses during admission (Additional file 1: Table S1). Cognitive function was classified as normal or impaired. The following conditions were considered impaired cognition: dementia, mild cognitive impairment, intellectual disability, and/or delirium at time of admission. Reasons for the index admission were categorized as: gastro-intestinal, infection, malignancy, inflammatory, (auto-) intoxication, renal and/or electrolyte problems, allergy/anaphylaxis, syncope, cardiac, and other. Day of discharge was divided 
into weekday (Monday till Friday) or weekend day (Saturday and Sunday). Season of discharge was classified as: summer (June, July, August), autumn (September, October, November), winter (December, January, February), and spring (March, April, May). Information about readmissions was obtained by checking the electronic patient record system for admissions.

All index admissions were categorized as being caused by an $\mathrm{AE}$ or not. To define an $\mathrm{AE}$, we used the following definition of the Dutch Internal Medicine Association: 'Any event or state during or following treatment by a specialist or a general practitioner that influenced the health of the patient in such way that renewed treatment was necessary or that it led to damage' [20]. Admissions because of problems we considered resulting from the progression of disease were not considered AEs. This definition is comparable to the definition used in other studies investigating AEs [21, 22]. AEs were divided into the following categories: medication-related, chemotherapyrelated, diabetes mellitus-related, procedure-related, and other. To evaluate whether or not the admission was truly based on an AE, two independent researchers (FM and LS) evaluated the admission reason. In case of disagreement, a third independent researcher (EP or PS) [1, 23] decided on the issue. The four researchers followed an E-learning course on the identification of AEs. The group of patients with an index admission because of an AE will further be referred to as AE group.

A readmission was defined as an unplanned admission through the ED to the department of internal medicine within 30 days after discharge. The reasons for readmission were categorized in the same way as the reasons for index admission. The no readmission group consisted of the patients who were not readmitted within 30 days after discharge and who were alive.

\section{Statistical analysis}

To investigate the factors that are associated with a readmission, we compared the readmission group with the no readmission group in the total study population (all ages), and in both younger and older patients. The same analyses were performed in the subgroup of patients with an index admission because of an AE. SPSS Statistics for Windows version 22.0 (SPSS Inc., Chicago, Illinois) was used to analyse all data. Data were shown as medians with ranges or numbers with percentages and odds ratios (ORs) with $95 \%$ confidence intervals (95\% CI). Inter-group differences were compared using the Chisquare test or Fisher's exact test for categorical data and the Mann-Whitney U test for continuous data. Cohen's kappa was used to calculate the inter-rater agreement concerning AEs as reason for admission.
To evaluate which factors were related to readmission, we performed univariate and multivariate logistic regression analyses. Due to insufficient cases per admission reason category, this factor could not be included in the multivariate analyses. In addition, IADL was omitted out of the equation due to collinearity with ADL. In the multivariate analyses in the $\mathrm{AE}$ group of younger patients, cognition and living situation could not be included in the analyses due to insufficient cases. Age was included as a dichotomous variable ( $\leq 65$ years (reference) vs. $>65$ years) in the analyses of the total study population. In the multivariate analyses in younger and older patients, age was included as a linear variable. CCI was included as a dichotomous variable: score of $0-2$ (reference) vs. $>2$. The number of medications was categorized as follows: $0-5$ (reference), $5-10,10-15$, and $>15$ medications. For season of discharge, we used summer as reference, for day of discharge, weekday was reference, and for sex, female was reference. $p$ values below 0.05 were considered statistically significant.

The MUMC + Medical Ethics Committee approved this study.

\section{Results}

\section{Total study population}

In the study period, there were 940 index admissions (940 patients, Fig. 1). The readmission rate was $17.3 \%$ and 72 patients $(44.2 \%)$ were readmitted for the same reason as their index admission. The median number of days until readmission was 10 (range $0-30$, Table 1 ).

In the readmission group, CCI was significantly higher (due to a skewed distribution, $p=0.04$ ) and patients more often had an index admission because of a malignancy ( 33.1 vs. $14.5 \%, p<0.001$ ) and were less often admitted because of an (auto-)intoxication (0.6 vs. $8.5 \%, p<0.001)$. Furthermore, patients discharged during autumn were significantly less often readmitted $(p=0.02)$ than when discharged during another season (Table 1). Index admissions because of an $\mathrm{AE}$ were equally prevalent in the readmission and the no readmission group.

Multivariate analyses in our total study population revealed that only ADL dependency was associated with readmissions (OR 2.43, $p=0.03$ ) (Table 2).

\section{Younger and older patients}

Of the younger patients, $16.9 \%(n=82)$ were readmitted after median 9 days. Age (56 vs. 50 years, $p=0.001$ ) and CCI ( 2 vs. $1, p=0.005)$ were significantly higher in the readmission group than in the no readmission group (Table 1). In the readmission group, patients more often had a malignancy (34.1 vs. $14.6 \%, p<0.001)$, but less 


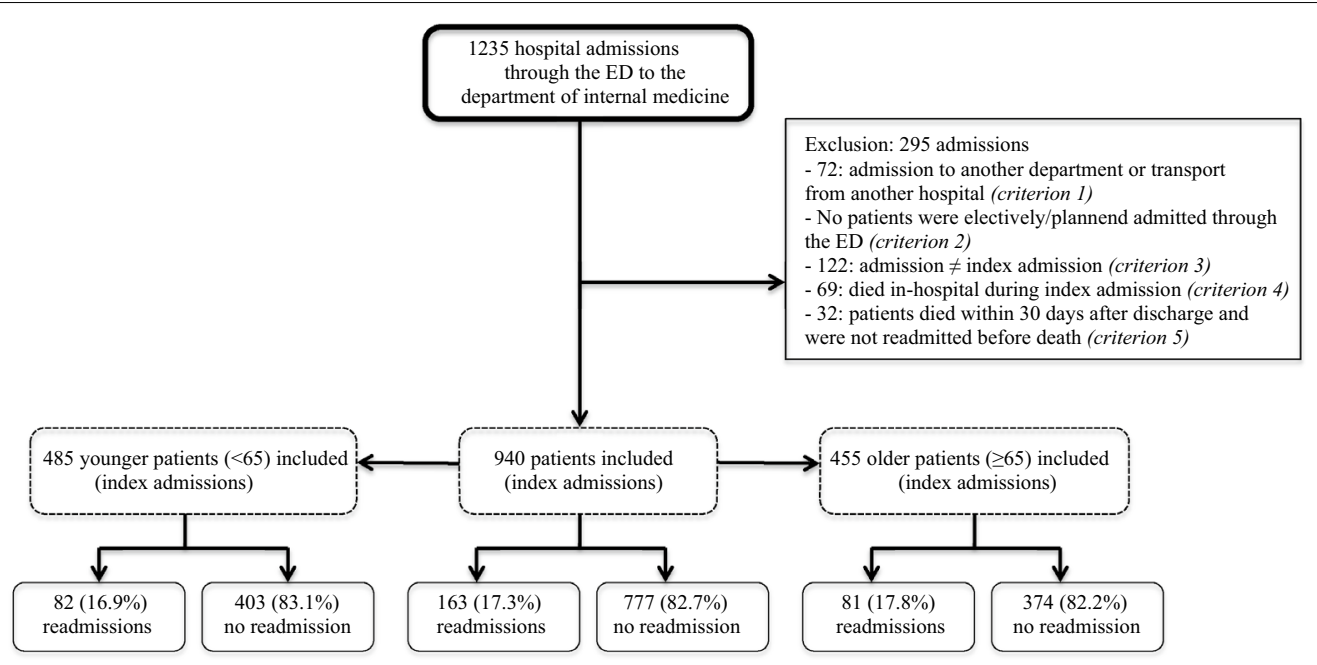

Fig. 1 Flow chart. ED emergency department

often an (auto)-intoxication (1.2 vs. $15.9 \%, p<0.001)$ as reason for the index admission.

Multivariate analyses showed that age was associated with the early readmissions (OR 1.03 per year) (Table 2).

Of the older patients, $17.8 \%(n=81)$ were readmitted after median 11 days. In the readmission group, patients used fewer medications (median 5 vs. 7, $p=0.006$ ), more often had a malignancy as reason for the index admission (32.1 vs. $14.4 \%, p<0.001$ ), and were less often discharged during autumn ( 11.1 vs. $26.5 \%, p=0.002)$.

Multivariate analyses in older patients showed that ADL dependency was associated with readmissions (OR 3.19) (Table 2). Patients using 10-15 medications (OR 0.40 ), and being discharged during autumn (OR 0.33) were significantly less often readmitted.

\section{Patients with an index admission because of an $\mathrm{AE}$}

In the AE group (of all patients, $35.4 \%$ of the study population), 65 patients $(19.5 \%)$ were readmitted compared with 98 patients $(16.1 \%, p=0.19$, data not shown) in the non-AE group. In the $\mathrm{AE}$ group, 29 patients (44.6\%) were readmitted because of the same reason as the index admission.

Patients in the readmission group less often had a medication-related $\mathrm{AE}$, and more often a chemotherapyrelated or a procedure-related $\mathrm{AE}$ than those in the no readmission group (Table 3). The inter-rater agreement for the judgment whether or not the admission was due to a healthcare-related AE was high (Cohen's kappa: 0.82 ).

Multivariate analyses of the $\mathrm{AE}$ group revealed that being ADL dependent was associated with readmissions (OR 7.05, Table 2).
Of the younger patients with an index admission because of an $\mathrm{AE}(n=157), 33(21.0 \%)$ patients were readmitted. Of these, 17 (51.5\%) were readmitted for the same reason. Patients in the readmission group less often had a medication-related $\mathrm{AE}$, and more often a chemotherapy-related $\mathrm{AE}$ than those in the no readmission group (Table 3).

In the multivariate analyses of the $\mathrm{AE}$ group of younger patients, no factors were found to be associated with readmissions (Table 2).

Of the older patients with an index admission because of an $\operatorname{AE}(n=176), 32$ patients $(18.2 \%)$ were readmitted, of whom $12(37.5 \%)$ were readmitted for the same reason. No statistically significant differences in the prevalence of the categories of AEs were found between the readmission and the no readmission group (Table 3).

Multivariate analyses in this AE group showed that ADL dependency was associated with readmissions (OR 10.0) (Table 2).

\section{Discussion}

We found that $17.3 \%(n=163)$ of the medical patients admitted through the ED were readmitted within 30 days. ADL dependency was associated with readmissions in patients of all ages and in older patients (OR 2.43 and OR 3.19, respectively). Insightful inter-group differences were also found. In the readmission group of all ages and younger patients, CCI scores were higher than in the no readmission group. In both groups, more patients were readmitted when their index admission was due to a malignancy. Interestingly, being admitted because of an (auto-)intoxication was associated with fewer readmissions in all ages and in younger patients. No association 


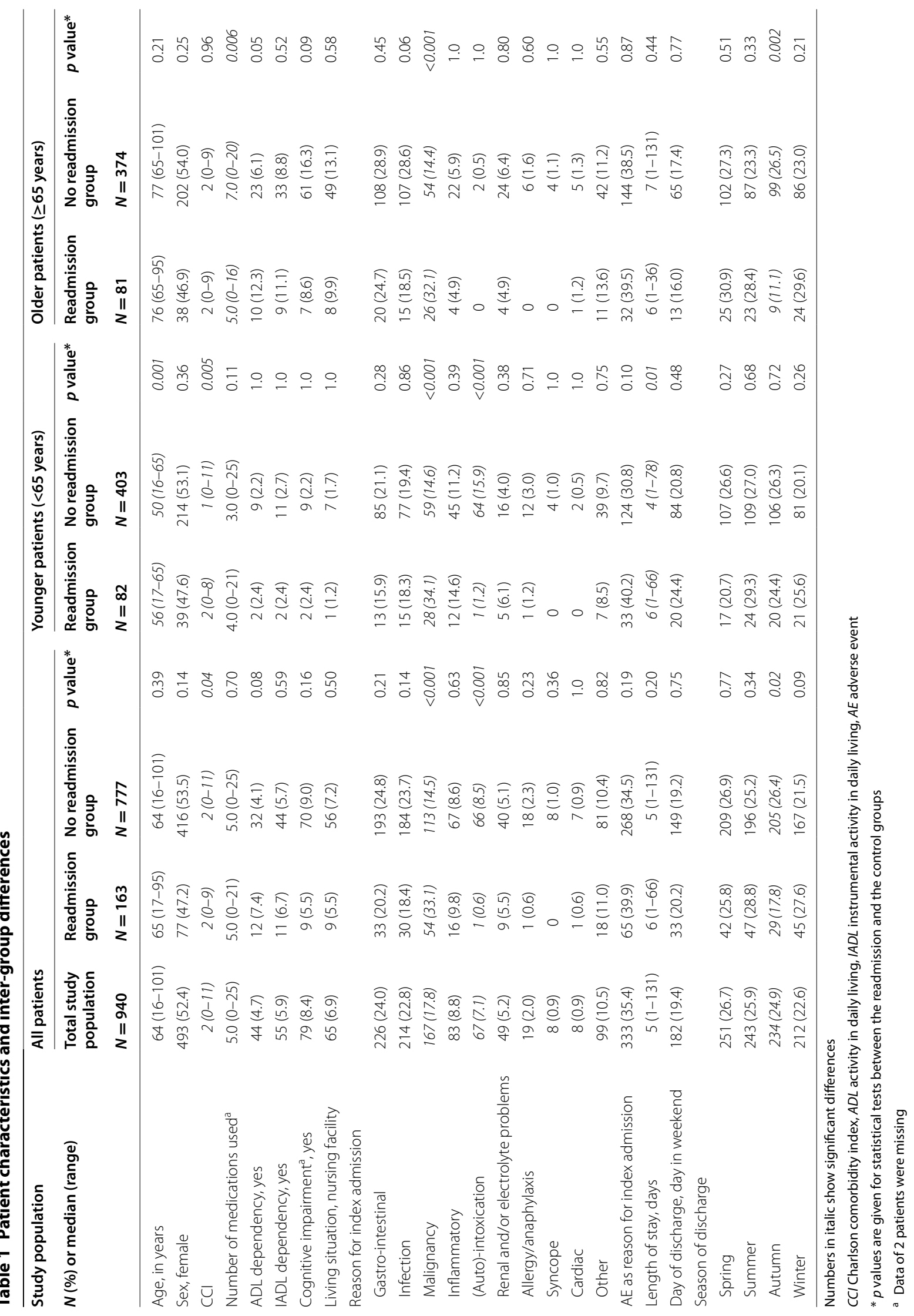




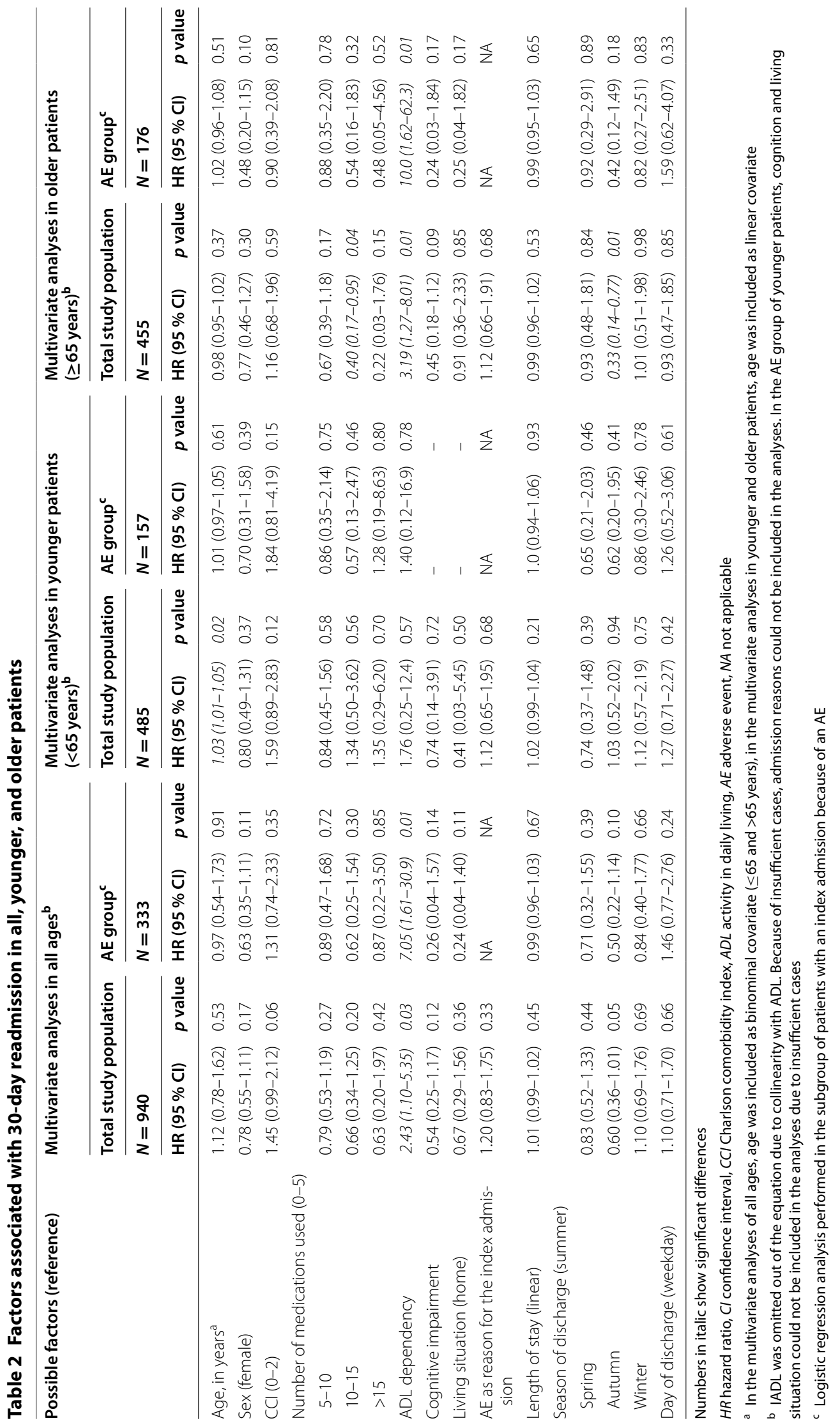




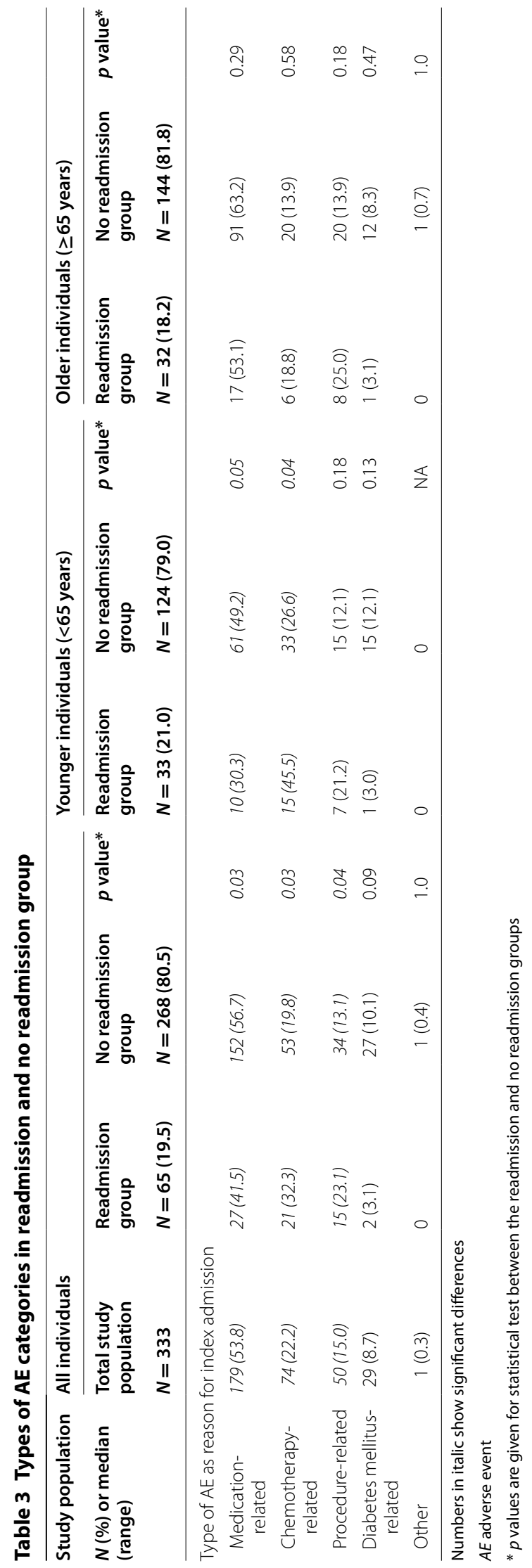


was found between index admissions because of an $\mathrm{AE}$ and readmissions.

\section{Total study population}

The 30-day readmission rate we found (17.3\%) is comparable with that of two other European studies [9, 24] and, despite of the differences in healthcare organization and insurance, to Asian $[4,25]$ and American studies [1, $3,5,8,26]$. This could indicate that the reasons for readmission lie in underlying diseases and/or patient characteristics rather than in the organization of the healthcare system. This hypothesis is supported by our and others' findings that patients who were admitted because of a malignancy were more likely to be readmitted $[4,25]$.

ADL dependency was independently associated with readmissions, which was also shown in one other study [26], but not in another [3]. Furthermore, in the intergroup analysis, we, like others [3, 4], found CCI scores to be higher in the readmission than in the no readmission group. However, as CCI was not associated with readmissions in our multivariate analyses and as the association with ADL dependency was not a consistent finding in the literature, other factors could be associated with readmissions and should be investigated. Further, we could not confirm the finding in other studies that a longer length of stay $[1,3,4,8,26]$ is associated with more readmissions. Furthermore, we like others $[4,5,8]$ found no association between age and readmission, unlike others $[1,15,26]$. Finally, our hypothesis that patients with AEs are more vulnerable, and thus at higher risk of a hospital readmission could not be confirmed as admissions because of AEs were not associated with more readmissions.

\section{Younger and older patients}

Readmissions occurred equally often in younger and older patients (16.9 and $17.8 \%$, respectively). As we expected, different factors were associated with readmissions in the two age groups. In younger patients, age (OR 1.03 per year) was associated with readmissions, but we did not find age to be important in older patients. We hypothesize that after reaching a certain age, age per se is less important. In older patients, ADL dependency was associated with readmissions (OR 3.19), which is in line with the findings of another study [2]. Two factors were found to be associated with fewer readmissions: using more medications ( $10-15$ vs. $0-5$, OR 0.40$)$ and being discharged during autumn (OR 0.33). However, we only investigated 1 year and our finding could also be a 'finding by chance' and should thus be interpreted cautiously.

Until now, no factors are found to be consistently associated with readmissions, and therefore, it remains difficult to predict which patients are at risk for a readmission. However, our study suggests that interventions that aim to reduce readmissions should focus on older patients who are ADL dependent. In addition, as our study shows that readmissions occur just as often in younger, and in older patients, interventions should also focus on these younger patients, especially the 'oldest' younger patients.

\section{Subgroup of patients with an index admission because of an $\mathrm{AE}$}

In the $\mathrm{AE}$ group, patients were equally often readmitted as in the non-AE group. ADL dependency, again, was associated with readmissions (OR 7.05). Interestingly, patients less often had a medication-related $\mathrm{AE}$ in the readmission than in the no readmission group. An explanation could be that for medication-related AEs, a solution is available, for example, discontinuation of the drug or adjustment of dosage. This could make these patients less vulnerable for a readmission. On the other hand, patients more often had a chemotherapy-related $\mathrm{AE}$ in the readmission group than the no readmission group. Patients receiving chemotherapy often need prolonged and high-risk treatments, and are, therefore, more susceptible to readmissions. Finally, patients with procedure-related AEs leading to the index admission were more likely to be readmitted. We hypothesize that these patients are treated earlier, since specialists may be more cautious and readmit these patients more easily.

\section{Limitations}

This study has some limitations. First, due to the retrospective nature of this study, data collection depended on documentation in the electronic record. However, this record includes not only medical, but nursing information as well, so little information is lost. Second, acute readmissions arranged through other ways than the ED were missed. However, in our hospital, almost all acute admissions take place through the ED. Furthermore, patients admitted to other hospitals are missed as well. However, the method used in this study is comparable with that of other studies $[1,4,5]$, all patients are instructed to return to our hospital when (new) complaints evolve and, from our experience, only a few patients will go to another hospital. Furthermore, patients readmitted to other departments than internal medicine were not discussed. However, we only found 3 patients who were readmitted to other departments. Therefore, it is unlikely that our results were influenced by these readmissions. Third, our sample size is relatively small. Therefore, this study has less power to detect certain associations, and thus, associations could have been missed. Fourth, we focused on acute admissions 
through the ED to the department of internal medicine. Future research should also focus on readmissions after planned admissions and/or investigate factors associated with readmissions to other departments than internal medicine.

\section{Conclusions}

Since readmissions are a serious burden to patients and considered an indicator of quality of care, it is important for hospitals to reduce readmissions. Moreover, as readmissions are common in medical patients, in both younger and older adult patients, they remain a great concern in terms of patient safety. We found that patients with a higher CCI or an index admission because of a malignancy, a chemotherapy-related $\mathrm{AE}$ or a procedurerelated $\mathrm{AE}$ were more likely to be readmitted. However, index admissions because of AEs were not more frequently followed by readmissions than index admissions for other reasons. Furthermore, being ADL dependent was found to be a factor related to readmissions in both all ages and older patients. Thus, care (including post-discharge care) should be tailored to specific patient's needs, with special attention to ADL dependency.

\section{Additional file}

Additional file 1: Table S1. (Part of) Standard information obtained by nurses at admission.

\begin{abstract}
Abbreviations
ADL: activities of daily living; $A E(s)$ : adverse event(s); CCl: Charlson comorbidity index; Cl: confidence interval; ED: emergency department; GP: general practitioner; IADL: instrumental activities of daily living; MUMC+: Maastricht University Medical Centre; OR: odds ratio.
\end{abstract}

\section{Authors' contributions}

FJHM: acquired data and drafted the manuscript. LS: acquired data and contributed to the revision of the manuscript. EP: contributed substantially to its revision. CDAS: contributed substantially to its revision. PMS: contributed substantially to its revision and takes responsibility for the paper as a whole. All authors read and approved the final manuscript.

\section{Author details \\ ${ }^{1}$ Division of General Medicine, Department of Internal Medicine, Maas- tricht University Medical Centre, Maastricht University, PO Box 5800, 6202 AZ Maastricht, The Netherlands. ${ }^{2}$ Section Clinical Geriatric Medicine, Division of General Medicine, Department of Internal Medicine, Maastricht University Medical Centre, Maastricht University, Maastricht, The Netherlands. ${ }^{3}$ Division of General Medicine, Department of Internal Medicine, and Cardiovascular Research Institute Maastricht, Maastricht University Medical Centre, Maastricht University, Maastricht, The Netherlands. ${ }^{4}$ Section of Acute Medicine, Division of General Medicine, Department of Internal Medicine, School of CAPHRI, Maastricht University Medical Centre, Maastricht University, Maastricht, The Netherlands.}

\section{Competing interests}

The authors declare that they have no competing interests.

\section{Ethics approval and consent to participate}

The MUMC + Medical Ethics Committee approved this study. Consent to participate was not applicable.
Received: 10 February 2016 Accepted: 7 September 2016

Published online: 15 September 2016

\section{References}

1. Park L, Andrade D, Mastey A, Sun J, Hicks L. Institution specific risk factors for 30 day readmission at a community hospital: a retrospective observational study. BMC Health Serv Res. 2014;14:40.

2. Caplan GA, Brown A, Croker WD, Doolan J. Risk of admission within 4 weeks of discharge of elderly patients from the emergency department-the DEED study. Discharge of elderly from emergency department. Age Ageing. 1998:27(6):697-702.

3. Hasan O, Meltzer DO, Shaykevich SA, Bell CM, Kaboli PJ, Auerbach AD, et al. Hospital readmission in general medicine patients: a prediction model. J Gen Intern Med. 2010;25(3):211-9.

4. Shu CC, Lin YF, Hsu NC, Ko WJ. Risk factors for 30-day readmission in general medical patients admitted from the emergency department: a single centre study. Intern Med J. 2012;42(6):677-82.

5. Moran J, Colbert CY, Song J, Hull J, Rajan S, Varghees S, et al. Residents examine factors associated with 30-day, same-cause hospital readmissions on an internal medicine service. Am J Med Qual. 2013;28(6):492-501.

6. Fischer C, Anema HA, Klazinga NS. The validity of indicators for assessing quality of care: a review of the European literature on hospital readmission rate. Eur J Pub Health. 2012;22(4):484-91.

7. Ashton CM, Wray NP. A conceptual framework for the study of early readmission as an indicator of quality of care. Soc Sci Med. 1996:43(11):1533-41.

8. Allaudeen N, Vidyarthi A, Maselli J, Auerbach A. Redefining readmission risk factors for general medicine patients. J Hosp Med. 2011;6(2):54-60.

9. Fabbian F, Boccafogli A, De Giorgi A, Pala M, Salmi R, Melandri R, et al. The crucial factor of hospital readmissions: a retrospective cohort study of patients evaluated in the emergency department and admitted to the department of medicine of a general hospital in Italy. Eur J Med Res. 2015;20(1):6.

10. Thomas EJ, Brennan TA. Incidence and types of preventable adverse events in elderly patients: population based review of medical records. BMJ. 2000;320(7237):741-4.

11. Onder G, Pedone C, Landi F, Cesari M, Della Vedova C, Bernabei R, et al. Adverse drug reactions as cause of hospital admissions: results from the Italian Group of Pharmacoepidemiology in the Elderly (GIFA). J Am Geriatr Soc. 2002;50(12):1962-8.

12. Magdelijns FJ, Pijpers E, Rouhl RP, Hannemann PF, Ten Broeke RH, Dings JT, et al. Acute hospital admissions because of health care-related adverse events: a retrospective study of 5 specialist departments. J Am Med Dir Assoc. 2015;16:1055-61.

13. Magdelijns FJ, Gulikers D, Pijpers E, Koopmans RP, Stassen PM. Registering complications at admission via the emergency department: an opportunity for improvement. Neth J Med. 2013;71(1):44-9.

14. Alvarez PA, Bril F, Castro V, Meiville I, Gonzalez CD, Centurion IG, et al. Adverse drug reactions as a reason for admission to an internal medicine ward in Argentina. Int J Risk Saf Med. 2013;25(3):185-92.

15. Pirmohamed M, James S, Meakin S, Green C, Scott AK, Walley TJ, et al. Adverse drug reactions as cause of admission to hospital: prospective analysis of 18820 patients. BMJ. 2004;329(7456):15-9.

16. Pouyanne P, Haramburu F, Imbs JL, Begaud B. Admissions to hospital caused by adverse drug reactions: cross sectional incidence study. French Pharmacovigilance Centres. BMJ. 2000:320(7241):1036.

17. Thijssen WA, Giesen PH, Wensing M. Emerg departments in The Netherlands. Emerg Med J. 2012;29(1):6-9.

18. Charlson ME, Pompei P, Ales KL, MacKenzie CR. A new method of classifying prognostic comorbidity in longitudinal studies: development and validation. J Chronic Dis. 1987:40(5):373-83.

19. Ramiarina RA, Ramiarina BL, Almeida RM, Pereira WC. Comorbidity adjustment index for the international classification of diseases, 10th revision. Rev Saude Publica. 2008;42(4):590-7.

20. Nederlandse Internisten Vereniging (NIV); Complicatieregistratie (E-learning). The Netherlands. http://www.internisten.nl/home/kwaliteit/ complicatieregistratie/complicatieregistratie. 
21. Zegers M, de Bruijne MC, Wagner C, Groenewegen PP, Waaijman R, van der Wal G. Design of a retrospective patient record study on the occurrence of adverse events among patients in Dutch hospitals. BMC Health Serv Res. 2007;7:27.

22. Baines R, Langelaan M, de Bruijne M, Spreeuwenberg P, Wagner C. How effective are patient safety initiatives? A retrospective patient record review study of changes to patient safety over time. BMJ Qual Saf. 2015;24(9):561-71.

23. Leppin AL, Gionfriddo MR, Kessler M, Brito JP, Mair FS, Gallacher K, et al. Preventing 30-day hospital readmissions: a systematic review and metaanalysis of randomized trials. JAMA Intern Med. 2014;174(7):1095-107.
24. Maurer PP, Ballmer PE. Hospital readmissions - are they predictable and avoidable? Swiss Med Wkly. 2004;134(41-42):606-11.

25. Bisharat N, Handler C, Schwartz N. Readmissions to medical wards: analysis of demographic and socio-medical factors. Eur J Intern Med. 2012;23(5):457-60.

26. Jencks SF, Williams MV, Coleman EA. Rehospitalizations among patients in the Medicare fee-for-service program. N Engl J Med. 2009;360(14):1418-28.

\section{Submit your next manuscript to BioMed Central and we will help you at every step:}

- We accept pre-submission inquiries

- Our selector tool helps you to find the most relevant journal

- We provide round the clock customer support

- Convenient online submission

- Thorough peer review

- Inclusion in PubMed and all major indexing services

- Maximum visibility for your research

Submit your manuscript at

www.biomedcentral com/submit 